\title{
Trehalose/hyaluronate eyedrop effects on ocular surface inflammatory markers and mucin expression in dry eye patients
}

This article was published in the following Dove Press journal:

Clinical Ophthalmology

\section{Chiara Fariselli \\ Giuseppe Giannaccare \\ Michela Fresina \\ Piera Versura}

Ophthalmology Unit, Department of Experimental, Diagnostic and Specialty Medicine, School of Medicine (DIMES), Alma Mater Studiorum University of Bologna and St Orsola-Malpighi Teaching Hospital, Bologna, Italy
Correspondence: Piera Versura Ophthalmology Unit, DIMES University of Bologna, St Orsola-Malpighi Hospital, Pad I Palagi, Via Palagi 9, Bologna 40I38, Italy

$\mathrm{Tel}+39512142850$

Fax +39 5I 34282 I

Email piera.versura@unibo.it
Aim: To assess the ocular surface parameters, inflammatory marker level in tears, and mucin expression in conjunctival epithelium before and after treatment with trehalose/hyaluronate tear substitute in dry eye (DE) patients.

Patients and methods: Fifteen DE patients were evaluated in an open-label, pilot study at enrollment, after 2 days of washout (baseline) and after 1 and 2 months (endpoint) of treatment with a trehalose/hyaluronate tear substitute (one drop/eye/three times daily). Data for symptoms of discomfort (Ocular Surface Disease Index and Visual Analogue Scale pain score), tear film (Schirmer test I, tear film breakup time), ocular surface damage (corneal National Eye Institute) and conjunctival van Bijsterveld scores, impression cytology scored by Nelson's grade and goblet cells (GCs) number $/ \mathrm{mm}^{2}$ analysis, and MUC4 immunostaining, and inflammation (interleukin [IL]-1 $\beta$, IL-6, and IL-8 levels) were measured.

Results: Significant changes at endpoint as compared to baseline were found for Ocular Surface Disease Index score (respectively, mean \pm SD, $22.2 \pm 2.9$ vs 38.7 \pm 12.7 ), Visual Analogue Scale score ( $3.4 \pm 1.3$ vs $6.6 \pm 1.4$ ), tear film breakup time ( $8.6 \pm 1.28$ vs $6.17 \pm 1.9$ seconds), corneal staining (National Eye Institute grade $1.23 \pm 0.64$ vs $3.37 \pm 0.49)$, conjunctival staining $(1.73 \pm 1.14$ vs $4.17 \pm 0.91$ ), impression cytology (Nelson grade $1.10 \pm 0.20$ vs $1.63 \pm 0.54$ ), and GC density $\left(139.9 \pm 22.0\right.$ vs $\left.107.8 \pm 16.2 \mathrm{GC} / \mathrm{mm}^{2}\right)$. IL-1 $\beta$, IL-6, and IL-8 tear levels showed a significant decrease at endpoint as compared to baseline (respectively, $\mathrm{pg} / \mathrm{mL}$ tears: $12.3 \pm 6.9,26.6 \pm 25.2$, $743.5 \pm 477.7$ vs $33.6 \pm 17.3,112.0 \pm 24.3,1,139.2 \pm 671.7)$.

Conclusions: A decrease in ocular discomfort symptoms, surface damage, and tear cytokine levels was shown after 2 months' treatment with trehalose/hyaluronate tear substitute in DE patients, along with a significant GC density recovery. These results may be associated with the synergic action of both trehalose and hyaluronic acid in targeting different entries of the DE vicious loop.

Keywords: dry eye, trehalose, hyaluronic acid, tear substitute, inflammation

\section{Introduction}

Dry eye disease (DED) is a widespread disease often associated with clinically significant ocular surface inflammation. It is defined as "a multifactorial disease of the ocular surface characterized by a loss of homeostasis of the tear film and accompanied by ocular symptoms, in which tear film instability and hyperosmolarity, ocular surface inflammation and damage, and neurosensory abnormalities play etiological roles". ${ }^{1}$ These key elements contribute to the DED pathophysiological process and are recognized as etiological triggers of a vicious circle, which is self-sustained without an appropriate therapeutic intervention. The DEWS I classification scheme presented two 
different types of DED, the aqueous-deficient dry eye (DE) and the evaporative DE; ${ }^{2}$ but the TFOS DEWS II identified DED subcategories, attributable to a "aqueous deficient and evaporative continuum", with signs of both reduced tear volume and deficient lipid layer.

The composition of the tear film and any change in tear profile give considerable information about eye health, as it is recognized that tear fluid can be used as a source of disease biomarkers $^{3}$ and tear biomarkers can be used to monitor the disease progression and the efficacy of a treatment. In DE, tear proteomic analysis can identify proteins, cytokines, and chemokines related to the severity of the disease., ${ }^{4,5}$ The presence of an inflammatory condition can be detected by the analysis of epithelial-derived inflammatory mediators, such as interleukin (IL)-1 $\beta$, IL-6, and IL-8. ${ }^{3,6,7}$ Identifying the level of ocular surface inflammation may guide therapeutic decisions ${ }^{8}$ allowing for more predictive and personalized therapies. $^{3}$

The first-line therapy in DED is the administration of tear substitutes, with the aim to restore the tear film deficient in quality and/or quality, ${ }^{9}$ and latest generation products are designed with combined formulas, targeted to address and break specific points within the DED vicious cycle. A new topical treatment composed of the novel promising combination between the natural disaccharide trehalose and the sodium hyaluronate has been quite recently commercialized, and it was shown to increase the tear film thickness in patients with mild to moderate DED, ${ }^{10}$ with an improvement in ocular surface parameters and patient satisfaction. ${ }^{11}$ The purpose of this study was to investigate whether an effect on other components of the DED vicious cycle may be seen in patients suffering from DED and treated with this trehalose/ hyaluronate combination and in particular on mucin expression and inflammatory markers in tears.

\section{Patients and methods}

This was a prospective, open-label, noncomparative, single-center pilot study performed at one research site (the Ophthalmic Unit, Alma Mater Studiorum University of Bologna, S. Orsola-Malpighi Teaching Hospital). The study was approved by the local Independent Ethics Committee of the S. Orsola-Malpighi Hospital and was conducted in accordance with the ethical principles of the Declaration of Helsinki and in agreement with the current legislation on clinical research in Italy. Signed informed consent was obtained from all individual participants included in the study. Fifteen patients (14 women aged 54 years as a median, one man aged 58 years) with subjective ocular discomfort and diagnosed with hyperevaporative DED were enrolled. Inclusion criteria were as follows: age higher than 18 years, good general health, ocular discomfort symptoms lasting $>3$ months, Ocular Surface Disease Index (OSDI) score $>18 / 100$, Schirmer test $>10 \mathrm{~mm} / 5$ minutes, tear film breakup time (TFBUT) $<10$ seconds, and moderate to severe corneal and conjunctival epithelial damage (National Eye Institute $[\mathrm{NEI}]$ score $>3$ ). Exclusion criteria were as follows: previous corneal surgery, contact lens wearers, concomitant ocular treatment, and known hypersensitivity to any component of the product under study.

Subjects who met the inclusion criteria were enrolled, and sodium chloride $0.9 \%$ sterile solution was dispensed to be used in both eyes for 2 days (washout period). This served to normalize the study population to an identical regimen of eyedrop use and allowed a minimization of any effect from previous lubricant eyedrop/medications use.

\section{Treatment}

The trehalose/hyaluronate tear substitute utilized in this study is marketed with the commercial name of Thealoz Duo $^{\circledR}$ (Thea Laboratoires, Clermont-Ferrand, France). It is a tear substitute composed of the combination of two ingredients: trehalose and sodium hyaluronate. The former is a natural alpha-linked disaccharide with high water retention capabilities. ${ }^{11}$ It is able to directly interact with nucleic acids, and it is implicated in anhydrobiosis, the ability of plants and animals to withstand prolonged periods of desiccation. The latter is the sodium salt of hyaluronic acid, an anionic glycosaminoglycan polysaccharide found in various connective tissue in humans. It has lubricant properties, forms a viscoelastic solution in water, and contributes to create a mechanical protection for cells. ${ }^{11}$ Patients were instructed to administer Thealoz Duo with the regimen of one drop/eye/ three times daily.

\section{Design of the study}

The study included four visits over 2 months period and specifically visit 0 ( $\mathrm{V} 0$, recruitment), after 1-3 days of washout (baseline, V1, beginning of treatment), after 1 month (V2), and after 2 months (endpoint, V3) of therapy. Patients were also invited to record any specific sensation at instillation.

\section{Tests}

The following tests were performed at all four visits:

Ocular discomfort symptoms were scored by the OSDI questionnaire. ${ }^{12}$ Factor analysis disclosed that there were three subscales, interpreted as subscale A, ocular symptoms 
(three questions); subscale B, vision-related function (six questions); and subscale $\mathrm{C}$, environmental triggers (three questions). Each score of the three subscales was calculated and included in the statistics. The total score ranges from 0 to 12 (no disability), 13-22 (light DE), 23-32 (moderate DE), and 33-100 (severe DE).

A Visual Analogue Scale (VAS) technique was used to measure symptom intensity. Patients were asked to mark the level of their discomfort on a $100 \mathrm{~mm}$, nonhatched VAS scale marked at one end as "no pain" and at the other as "pain as bad as it could be". Using a ruler, the score was determined by measuring the distance between the "no pain" end and the patient's mark, providing a range of scores from 0 to 100 with a higher score indicating greater pain intensity.

Schirmer test I was performed with validated sterile strips (ContaCare Ophthalmics and Diagnostics, Gujarat, India) placed in the inferior fornix of both eyes at the same time: pathological values were considered $<10 \mathrm{~mm}$ wetting after 5 minutes. ${ }^{13}$ TFBUT was carried out to evaluate tear film stability and was measured and recorded (average of three measurements) after instillation of $2 \mu \mathrm{L}$ unpreserved fluorescein sodium 2\% (galenic preparation from Fluoralfa $0.25 \%$, Alfa Intes, Casoria, NA, Italy). TFBUT was considered pathological with values $<10$ seconds. ${ }^{14}$

Corneal vital staining was performed to investigate the ocular surface damage by using fluorescein sodium $2 \%$, and staining details were enhanced using a 7,503 Boston yellow filter (equivalent to Kodak Wratten 12) and graded according to the NEI grading system (score $0-15) .{ }^{15}$ Conjunctival damage was graded on the basis of the conjunctival van Bijsterveld score (score 0-9). ${ }^{16}$

The following tests were performed at visits V1 and V3: Impression cytology was used to evaluate conjunctival epithelial metaplasia and goblet cell density (GD) and was scored by Nelson's grade ( $0-3)$. Goblet cell (GC) analysis was expressed in number of cells $/ \mathrm{mm}^{2} \cdot{ }^{17} \mathrm{MUC} 4$ is a mucin with lubricating, clearing, and barrier function, and its expression was analyzed with immunostaining (Ventana Medical System, Tucson, AZ, USA). The H-score (range: 0 [no staining in any cell] to 3 [all cells staining with the highest intensity]) was calculated as the summation of the product of staining intensity (range: $0-3$ ) and proportion of cells stained (range: $0-1 ; 0$, no cell stained and $1 \%-100 \%$, cell stained). ${ }^{18}$

The levels of three inflammatory cytokines IL-1 $\beta$, IL-6, and IL- 8 were detected by multiplex bead assay (Magnetic Luminex ${ }^{\circledR}$ Assay; Human Premixed Multi-Analyte Kit, R\&D Systems Europe, Ltd., Abingdon, UK), using a Luminex S-100 (Biorad Bio-Plex 100, Segrate Milano, Italy). Briefly, a minimum of $5 \mu \mathrm{L}$ tear samples were collected with a laboratory micropipette (Pipetman P, Gilson International B.V., Den Haag, the Netherlands) with a sterile tip positioned at the outer canthus. Five microliters of sample was diluted 1:5 with assay diluent; buffered protein with preservatives was incubated with antibody-coated capture beads for 2 hours at $22^{\circ} \mathrm{C}$. Washed beads were further incubated with biotin-labeled antihuman cytokine antibodies for 1 hour at $22^{\circ} \mathrm{C}$, followed by streptavidin phycoerythrin incubation. Standard curves of known concentrations of recombinant human cytokines were used to convert fluorescence units to cytokine concentrations $(\mathrm{pg} / \mathrm{mL})$.

\section{Satisfaction and tolerability}

Patient's satisfaction was evaluated at all four visits by a VAS-based satisfaction questionnaire composed of the following questions: 1) My eyes feel dry in the morning; 2) My eyes feel dry at the end of the day; 3) My eyes feel refreshed when I administer the product; 4) I frequently forgot my symptoms during the use of this product. Score at V0 and V1 referred to the feeling about the latest therapy administered prior to the beginning of the study. For statistical analysis, VAS score at baseline (V1) was compared with that recorded at endpoint (V3).

Tolerability was assessed by a VAS scoring of specific symptoms (blurring, redness, itching, and stinging) recorded upon instillation, and it was evaluated at all four visits as described above for satisfaction.

\section{Statistical analysis}

Data were statistically analyzed using the MedCalc 5.0 and SPSS software (IBM Corporation, Armonk, NY, USA) and applying Wilcoxon's test for paired data $(P<0.05$ was considered to be statistically significant). Descriptive statistics for tests and variables analyzed in subjects were reported as the mean \pm SD. Pearson's (r) correlation coefficients were applied when appropriate; correlations were considered statistically significant at $P<0.05$.

\section{Outcomes}

The outcomes for the mean change from baseline analyses for each subject were set on the $20 \%$ reduction for OSDI and VAS score, on the $20 \%$ increase for TFBUT, and on a one-point reduction for the corneal and conjunctival vital stain score.

\section{Results}

All subjects enrolled for the treatment completed the study. They declared to have administered the eye drops 
three times/day, as requested in the protocol. No significant changes in any of the tests performed were observed between enrollment (V0) and baseline visits (V1); data from visit V0 were included in graphs associated with V1.

All ocular parameters improved after the treatment, except for the Schirmer test values that did not change at endpoint as compared to baseline. Ocular subjective symptoms demonstrated a significant $(P<0.01)$ reduction of either OSDI and VAS score, and in particular, 14 of 15 patients were found to have a mean change from baseline reduced over $20 \%$ for both the measures. Analysis of the three subscales demonstrated a statistically significant change in the ocular discomfort in the vision-related function and environmental triggers subscales but not in ocular symptoms subscales (Figure 1).

A significant increase in TFBUT values was found at endpoint as compared to baseline (Figure 2) in all subjects, and in particular 11 of 15 patients were found to have a mean change from baseline increased over $20 \%$. Both the corneal and conjunctival damage scores were shown to be significantly reduced at endpoint as compared to baseline (Figure 2). For the NEI score, the mean change from baseline was reduced by one point in five patients and six showed a greater reduction by two points. For the van Bijsterveld score, the mean change from baseline was reduced by one point in three patients and seven showed a greater reduction by two points.

Impression cytology scores showed a statistically significant reduction and an improvement in GD at endpoint as compared to baseline (Figure 3). A light increase not statistically significant $(P=0.08)$ was demonstrated for MUC4 immunostaining at endpoint as compared to baseline, as the $\mathrm{H}$-score values were $1.2 \pm 0.4$ vs $1.1 \pm 0.5$, respectively.

IL-1 $\beta$, IL-6, and IL-8 tear levels were found above the minimum detectable level in all subjects at baseline with a significant $(P<0.01)$ decrease at endpoint as compared to baseline (Figure 4). Cytokine levels were correlated to surface damage at baseline (Pearson's r ranging 0.45-0.59, $P<0.0001$ ) but not at endpoint.
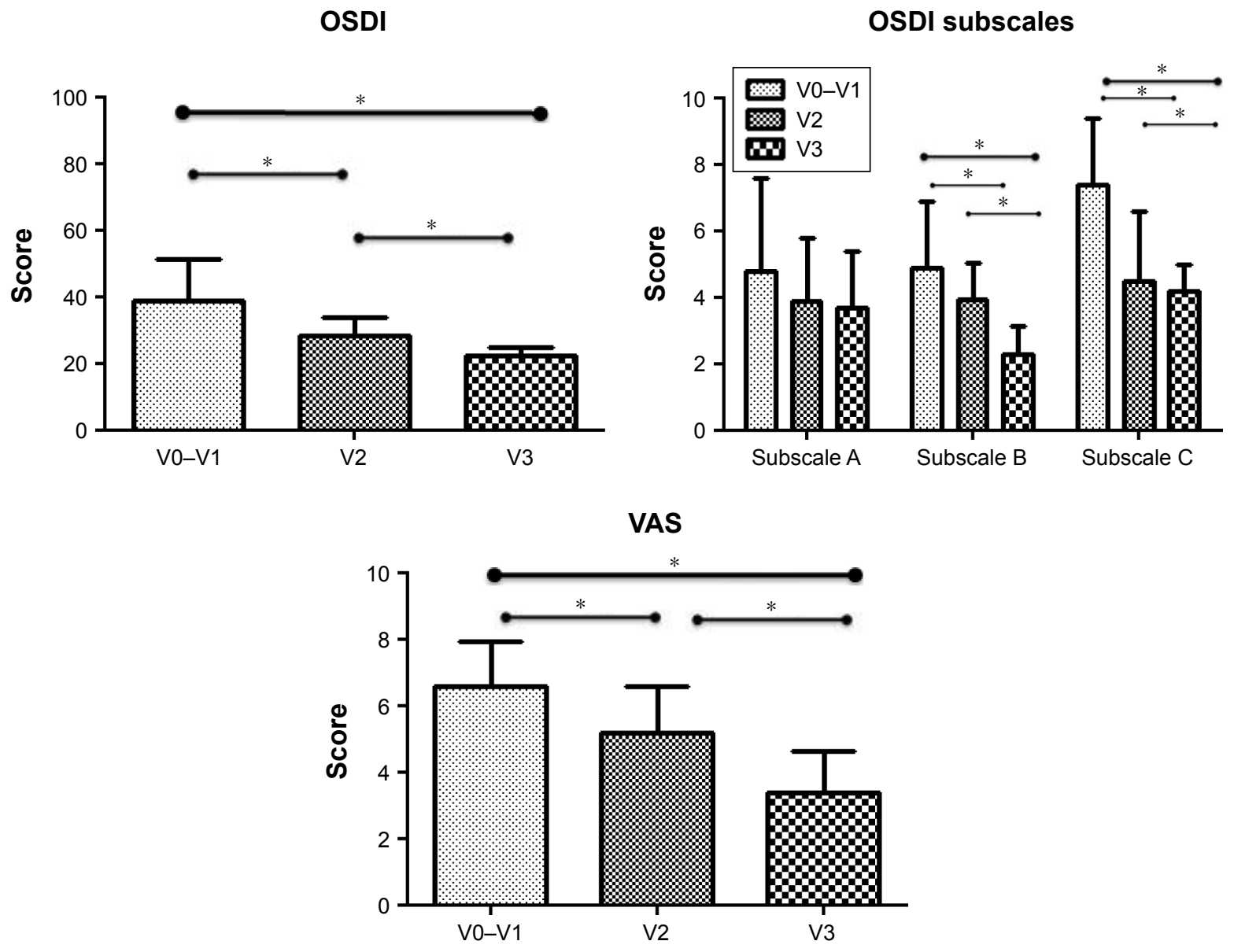

Figure I OSDI total score (upper left) and VAS scale (bottom) values are graphed. OSDI subscale values in the upper right are reported as subscale A (questions related to ocular symptoms), subscale $B$ (vision-related function), and subscale $C$ (environmental triggers).

Notes: *Significance $P<0.05$. V0, recruitment; VI, baseline; V2, after I month of treatment; V3, after 2 months of treatment (endpoint).

Abbreviations: OSDI, Ocular Surface Disease Index; VAS, Visual Analogue Scale. 


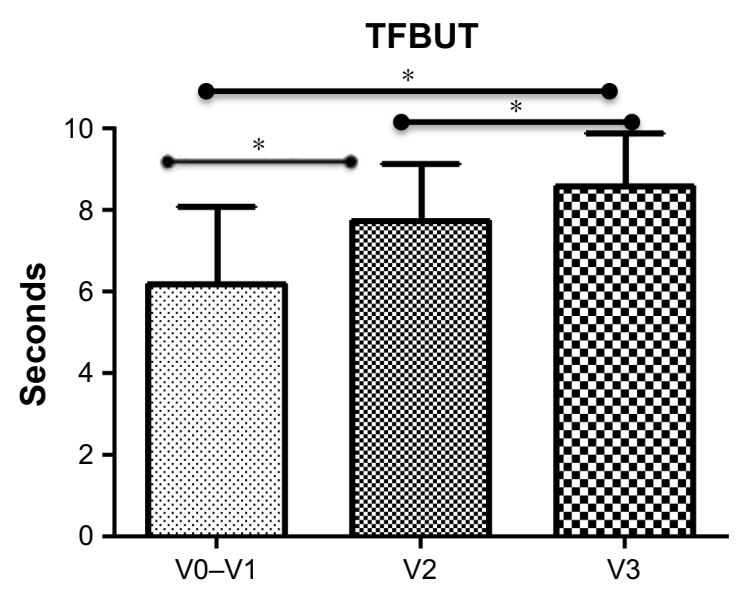

\section{Corneal NEI score}
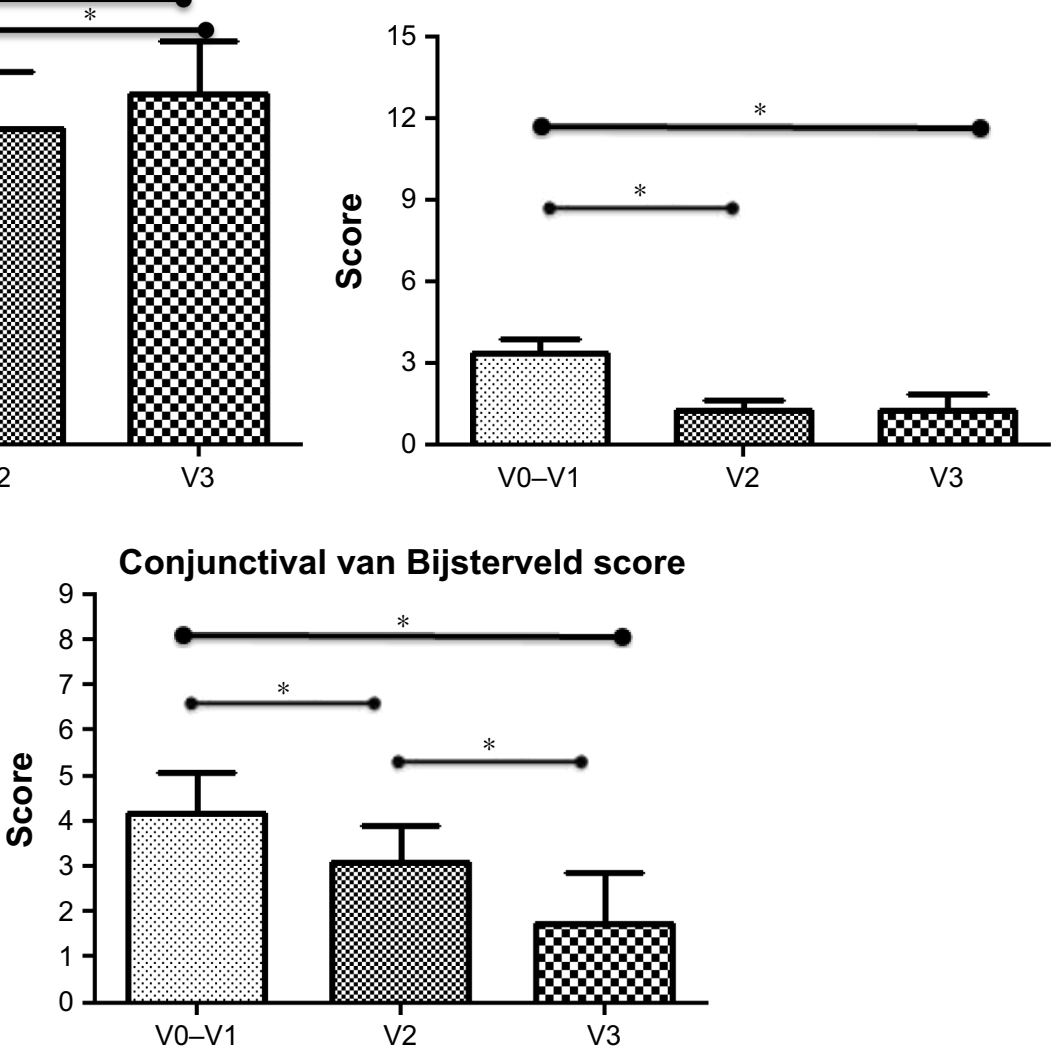

Figure 2 Tear stability TFBUT values (upper left), corneal damage scored by NEI score (upper right), and conjunctival damage scored by van Bijesterveld score (bottom) are graphed.

Notes: *Significance $P<0.05$. V0, recruitment; $\mathrm{VI}$, baseline; $\mathrm{V} 2$, after I month of treatment; $\mathrm{V} 3$, after 2 months of treatment (endpoint).

Abbreviations: NEI, National Eye Institute; TFBUT, tear film breakup time.

Results from the VAS-based satisfaction questionnaire are summarized in Table 1. All patients reported a significant decrease in the feeling of dryness both in the morning and at the end of the day, which was reduced by $50 \%$ at endpoint as compared to baseline. They also reported a feeling of refresh and the disappearance of their discomfort symptoms during the use of the product, with a VAS score improved. Only 2 of 15 patients had a light sensation of
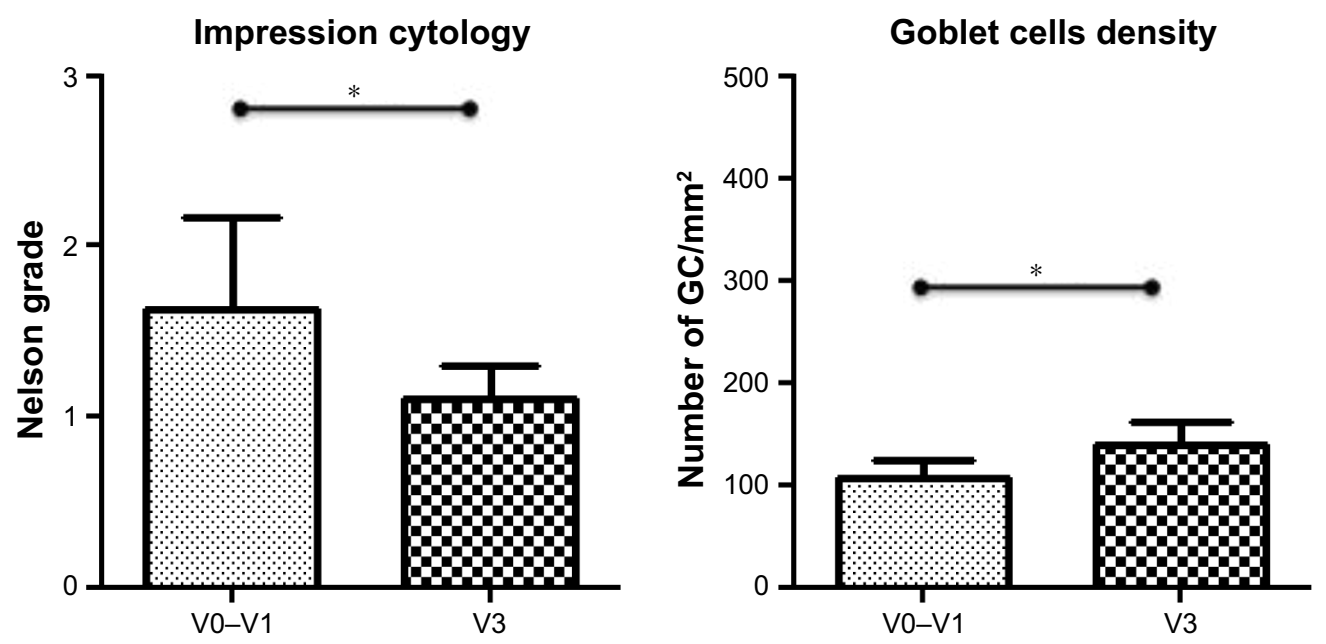

Figure 3 Conjunctival impression cytology scored by Nelson's grade (left side) and goblet cell (GC, right side) density expressed as number of GC/mm² are graphed, at baseline (V0-VI) and endpoint (V3).

Notes: *Significance $P<0.05$. V0, recruitment; VI, baseline; V2, after I month of treatment; V3, after 2 months of treatment (endpoint). 

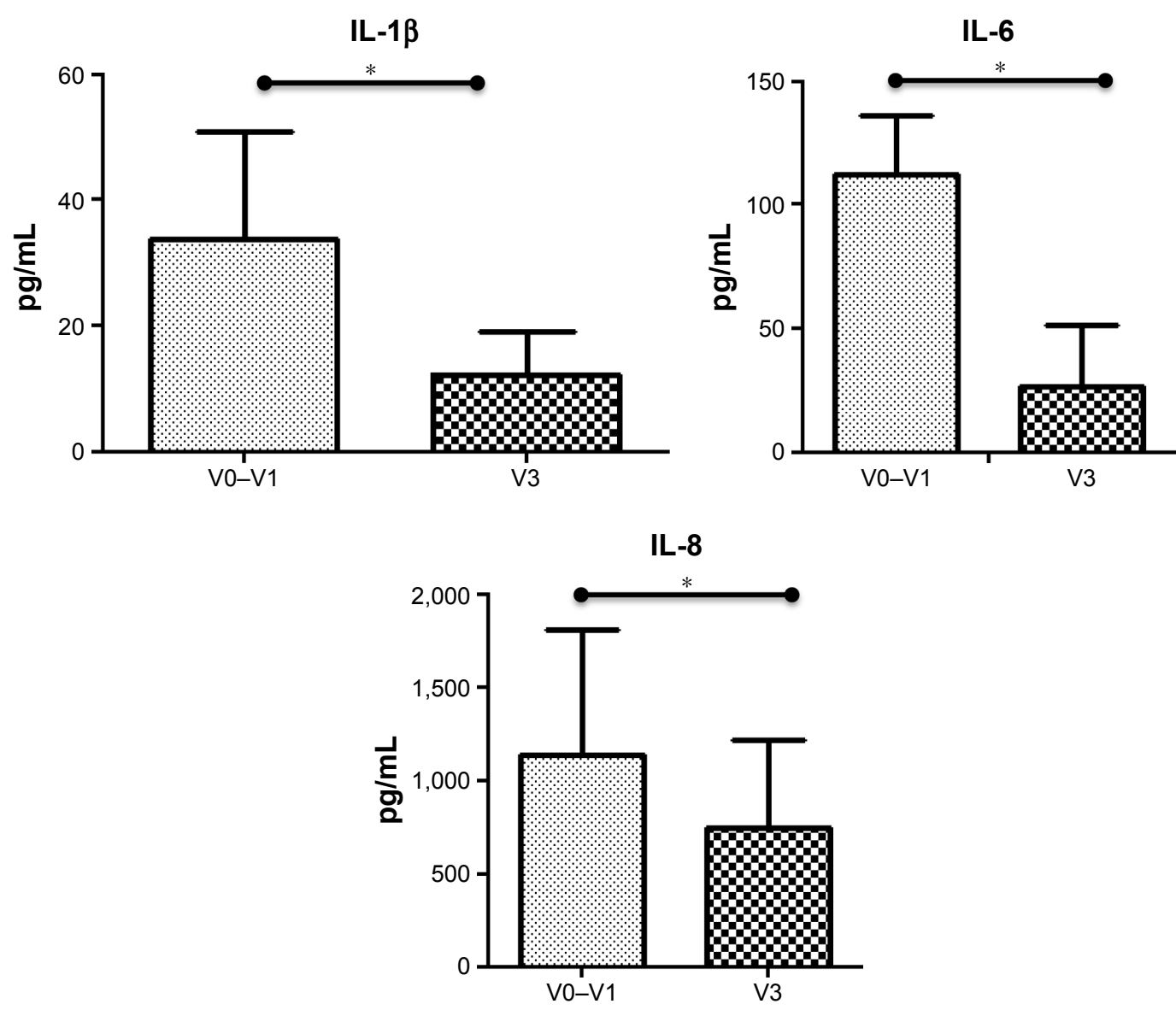

Figure 4 Tear levels of interleukin (IL)- I $\beta$ (upper left), IL-6 (upper right), and IL-8 (bottom) are graphed, at baseline (V0-VI) and endpoint (V3). Notes: *Significance $P<0.05$. V0, recruitment; VI, baseline; V2, after I month of treatment; V3, after 2 months of treatment (endpoint).

blurring, which disappeared after less than 3 minutes from instillation.

\section{Discussion}

The DE central core is characterized by a loop of cyclic events connecting tear film instability and hyperosmolarity, inflammatory response, and metaplastic changes of ocular surface epithelia, and any therapeutic approach is targeted to breakdown the loop at any point with the aim to prevent the disease progression.

Data from the present study in DE patients demonstrated an amelioration in ocular discomfort subjective symptoms, a decrease in ocular surface damage and tear cytokine levels, and an increase in tear film stability after 2 months' treatment with the association of trehalose/hyaluronic acid tear substitute.

As far as the reduction of the subjective symptoms of discomfort and ocular surface damage and the amelioration of tear stability after the treatment are concerned, our results are in agreement with a previous work, ${ }^{11}$ where, however, the trehalose/hyaluronate tear substitute was administered more frequently per day but only for 1 week. Interestingly, a reduction of a specific category of symptoms was shown after treatment, ie, discomfort related to the vision-related function and environmental triggers, which deserves further investigation concerning the effects of this treatment on the mechanisms of perception.

Table I Visual Analogue Scale-based satisfaction questionnaire results

\begin{tabular}{lllll}
\hline Question & V0-VI & V2 & V3 & Significance \\
\hline My eyes feel dry in the morning & $6.2 \pm I .6$ & $5.8 \pm 2.3$ & $3.8 \pm I .5$ & VI vs V2 \\
My eyes feel dry at the end of the day & $7.1 \pm I .8$ & $5.9 \pm 1.2$ & $3.3 \pm I .5$ & V2 vs V3 \\
My eyes feel refreshed when I administer the product & $2.5 \pm 1.5$ & $4.1 \pm 1.2$ & $6.3 \pm I . I$ & VI vs V3 \\
I frequently forgot my symptoms during the use of this product & $3.0 \pm 0.5$ & $4.3 \pm I .3$ & $5.1 \pm 0.9$ & Always P<0.0I \\
\hline
\end{tabular}

Notes: V0, recruitment; VI, baseline; V2, after I month of treatment; V3, after 2 months of treatment (endpoint). 
A significant improvement of conjunctival epithelium metaplasia was demonstrated after treatment associated with the recovery of the GC number but not of the transmembrane MUC4 expression. MUC4 plays a protective role in ocular surface epithelia, and its level was shown to be significantly lower in conjunctival epithelium of patients with DES compared with that in normal subjects. ${ }^{19}$ Our data do not support a mechanism of mucin induction taking place as a consequence of treatment, so far.

Clinically significant inflammation occurs in about half of patients with $\mathrm{DED},{ }^{8}$ playing an important role to initiate and perpetuate the disease.$^{20}$ Inflammation is demonstrated by the infiltration of T-cells in the conjunctiva and the presence of high levels of inflammatory mediators in the tears of symptomatic DED patients that shows a correlation with clinical disease parameters. ${ }^{21,22}$ IL-1, IL-6, and IL-8 levels are associated with the severity of the DED and correlate well with ocular surface parameters. ${ }^{23}$ Furthermore, the identification of DED patients with underlying inflammation may predict patient responses to treatment and help to find a more targeted clinical management. ${ }^{8,24}$ Latest tear substitute formulations include the so-called Multiple Action Tear Substitutes (MATS), ie, products with the purpose not only to alleviate symptoms but also to target the DED vicious loop. In this respect, some MATS have been designed with the purpose to potentially reduce the ocular surface inflammation, and several preclinical reports have been published. Some authors demonstrated the anti-inflammatory effects of tear substitutes in animal DE models, and in particular hyaluronic acid ${ }^{25-28}$ and omega-3 essentially fatty acid. ${ }^{29}$ The anti-inflammatory effects of different tear substitutes were compared in a rat model of corneal scraping-induced inflammation: the tear substitutes tested were able to reduce inflammatory cell infiltration in the corneal stroma, and one of them was also able to decrease the secretion of IL- 6 and IL-8. ${ }^{30}$ Topical application of a mixture of omega-3 essential fatty acids and hyaluronic acid improves clinical signs and decreases inflammatory cytokines on the ocular surface in a murine DED model. ${ }^{29}$ The expression of IL-1 $\beta$, IL-6, IL-8, and tumor necrosis factor (TNF)- $\alpha$ was reduced in in vitro inflammatory model of human corneal epithelial cells treated with drops containing a combination of epigallocatechin gallate and hyaluronic acid. The same topical treatment demonstrated decreased levels of IL-6, IL-8, and TNF- $\alpha$ in DE syndrome in rabbits. ${ }^{31}$

In human studies, the capability of three tear supplements (carboxymethylcellulose-glycerin-castor, carboxymethylcellulose, and hydroxypropyl guar) was demonstrated to reduce clinical markers of inflammation, and only the first was able to reduce DED-associated tear film inflammatory biomarkers. ${ }^{32}$ Our study showed a statistically significant decrease of specific cytokine levels in tears of DED patients treated with the association of trehalose/hyaluronic acid tear substitute.

This is the first pilot explorative study, and the main limitations consist of the small number of patients included and the lack of a comparative control group. Also, as DED is a progressive disease, time for evaluation longer than 2 months may be necessary to confirm the capability of this product in modulating the responses.

In conclusion, these results may be associated with the synergic action of both trehalose and hyaluronic acid in targeting different entries of the DE vicious loop; however, randomized studies are required to truly ascertain the magnitude of their clinical value.

\section{Acknowledgments}

Data from this study were presented in part at The Association for Research Invision and Opthalmology meeting 2017, Baltimore, MD, USA on May 7-11. The authors thank DrMarco Grillini, Department of Pathology, S. Orsola-Malpighi Hospital, Bologna for his valuable technical support.

\section{Disclosure}

The authors report no conflicts of interest in this work.

\section{References}

1. Craig JP, Nichols KK, Akpek EK, et al. TFOS DEWS II Definition and Classification Report. Ocul Surf. 2017;15(3):276-283.

2. [No authors listed] The definition and classification of dry eye disease: report of the Definition and Classification Subcommittee of the International Dry Eye WorkShop (2007). Ocul Surf. 2007;5(2):75-92.

3. Hagan S, Martin E, Enríquez-de-Salamanca A. Tear fluid biomarkers in ocular and systemic disease: potential use for predictive, preventive and personalised medicine. Epma J. 2016;7:15.

4. Chen W, Cao H, Lin J, Olsen N, Zheng SG. Biomarkers for primary Sjögren's syndrome. Genomics Proteomics Bioinformatics. 2015;13(4): 219-223.

5. Matheis N, Grus FH, Breitenfeld M, et al. Proteomics differentiate between thyroid-associated orbitopathy and dry eye syndrome. Invest Ophthalmol Vis Sci. 2015;56(4):2649-2656.

6. Hagan S, Tomlinson A. Tear fluid biomarker profiling: a review of multiplex bead analysis. Ocul Surf. 2013;11(4):219-235.

7. Enríquez-de-Salamanca A, Castellanos E, Stern ME, et al. Tear cytokine and chemokine analysis and clinical correlations in evaporative-type dry eye disease. Mol Vis. 2010;16:862-873.

8. Sambursky R. Presence or absence of ocular surface inflammation directs clinical and therapeutic management of dry eye. Clin Ophthalmol. 2016;10:2337-2343.

9. Jones L, Downie LE, Korb D, et al. TFOS DEWS II Management and Therapy Report. Ocul Surf. 2017;15(3):575-628.

10. Schmidl D, Schmetterer L, Witkowska KJ, et al. Tear film thickness after treatment with artificial tears in patients with moderate dry eye disease. Cornea. 2015;34(4):421-426. 
11. Pinto-Bonilla JC, del Olmo-Jimeno A, Llovet-Osuna F, HernándezGalilea E. A randomized crossover study comparing trehalose/ hyaluronate eyedrops and standard treatment: patient satisfaction in the treatment of dry eye syndrome. Ther Clin Risk Manag. 2015;11(11): 595-603.

12. Schiffman RM, Christianson MD, Jacobsen G, Hirsch JD, Reis BL. Reliability and validity of the Ocular Surface Disease Index. Arch Ophthalmol. 2000;118(5):615-621.

13. Monchy de I, Gendron G, Miceli C, Pogorzalek N, Mariette X, Labetoulle M. Combination of the Schirmer I and phenol red thread tests as a rescue strategy for diagnosis of ocular dryness associated with Sjogren's syndrome. Invest Ophthalmol Vis Sci. 2011;52:5167.e73.

14. Mengher LS, Bron AJ, Tonge SR, Gilbert DJ. A non-invasive instrument for clinical assessment of the pre-corneal tear film stability. Curr Eye Res. 1985;4(1):1-7.

15. Lemp MA. Report of the National Eye Institute/Industry workshop on Clinical Trials in Dry Eyes. Clao J. 1995;21(4):e32.

16. van Bijsterveld OP. Diagnostic tests in the Sicca syndrome. Arch Ophthalmol. 1969;82(1):10e4-14e4.

17. Mrugacz M, Kasacka I, Bakunowicz-Lazarczyk A, Kaczmarski M, Kulak W. Impression cytology of the conjunctival epithelial cells in patients with cystic fibrosis. Eye. 2008;22(9):1137-1140.

18. Kaur S, Momi N, Chakraborty S, et al. Altered expression of transmembrane mucins, MUC1 and MUC4, in bladder cancer: pathological implications in diagnosis. PLoS One. 2014;9(3):e92742.

19. Corrales RM, Narayanan S, Fernández I, et al. Ocular mucin gene expression levels as biomarkers for the diagnosis of dry eye syndrome. Invest Ophthalmol Vis Sci. 2011;52(11):8363-8369.

20. Pflugfelder SC, Jones D, Ji Z, Afonso A, Monroy D. Altered cytokine balance in the tear fluid and conjunctiva of patients with Sjogren's syndrome keratoconjunctivitis sicca. Curr Eye Res. 1999;19:201-211.

21. Lam H, Bleiden L, de Paiva CS, Farley W, Stern ME, Pflugfelder SC. Tear cytokine profiles in dysfunctional tear syndrome. Am JOphthalmol. 2009;147(2):198-205.

22. Boehm N, Riechardt AI, Wiegand M, Pfeiffer N, Grus FH. Proinflammatory cytokine profiling of tears from dry eye patients by means of antibody microarrays. Invest Ophthalmol Vis Sci. 2011;52(10):7725-7730.
23. Lam H, Bleiden L, de Paiva CS, Farley W, Stern ME, Pflugfelder SC. Tear cytokine profiles in dysfunctional tear syndrome. Am J Ophthalmol. 2009;147(2):198-205.

24. Pflugfelder SC. Antiinflammatory therapy for dry eye. Am JOphthalmol. 2004;137(2):337-342.

25. Vogel R, Crockett RS, Oden N, Laliberte TW, Molina L. Sodium Hyaluronate Ophthalmic Solution Study Group. Demonstration of efficacy in the treatment of dry eye disease with $0.18 \%$ sodium hyaluronate ophthalmic solution (vismed, rejena). Am J Ophthalmol. 2010;149(4): 594-601.

26. Chen W, Zhang X, Liu M, et al. Trehalose protects against ocular surface disorders in experimental murine dry eye through suppression of apoptosis. Exp Eye Res. 2009;89(3):311-318.

27. Cejková J, Ardan T, Cejka C, Luyckx J. Favorable effects of trehalose on the development of UVB-mediated antioxidant/pro-oxidant imbalance in the corneal epithelium, proinflammatory cytokine and matrix metalloproteinase induction, and heat shock protein 70 expression. Graefes Arch Clin Exp Ophthalmol. 2011;249(8):1185-1194.

28. Li J, Roubeix C, Wang Y, et al. Therapeutic efficacy of trehalose eye drops for treatment of murine dry eye induced by an intelligently controlled environmental system. Mol Vis. 2012;18:317-329.

29. Li Z, Choi J-H, Oh H-J, Park S-H, Lee J-B, Yoon KC. Effects of eye drops containing a mixture of omega-3 essential fatty acids and hyaluronic acid on the ocular surface in desiccating stress-induced Murine dry eye. Curr Eye Res. 2014;39(9):871-878.

30. Daull P, Feraille L, Elena PP, Garrigue JS. Comparison of the antiinflammatory effects of artificial tears in a rat model of corneal scraping. J Ocul Pharmacol Ther. 2016;32(2):109-118.

31. Tseng C-L, Hung Y-J, Chen Z-Y, Fang H-W, Chen K-H. Synergistic effect of artificial tears containing epigallocatechin gallate and hyaluronic acid for the treatment of rabbits with dry eye syndrome. PLoS One. 2016;11(6):e0157982.

32. Martin E, Oliver KM, Pearce EI, Tomlinson A, Simmons P, Hagan S. Effect of tear supplements on signs, symptoms and inflammatory markers in dry eye. Cytokine. 2018;105(105):37-44.
Clinical Ophthalmology

\section{Publish your work in this journal}

Clinical Ophthalmology is an international, peer-reviewed journal covering all subspecialties within ophthalmology. Key topics include: Optometry; Visual science; Pharmacology and drug therapy in eye diseases; Basic Sciences; Primary and Secondary eye care; Patient Safety and Quality of Care Improvements. This journal is indexed on Submit your manuscript here: http://www.dovepress.com/clinical-ophthalmology-journal

\section{Dovepress}

PubMed Central and CAS, and is the official journal of The Society of Clinical Ophthalmology (SCO). The manuscript management system is completely online and includes a very quick and fair peer-review system, which is all easy to use. Visit http://www.dovepress.com/ testimonials.php to read real quotes from published authors. 